\section{Cahiers de Narratologie}

Analyse et théorie narratives

Littérature et sciences

\title{
Affectivité et sentiment en économie politique : Cartas sobre los obstáculos que la naturaleza, la opinión y las leyes oponen a la felicidad pública du comte de Cabarrús (1795)
}

Marc Marti

\section{CpenEdition}

\section{Journals}

Édition électronique

URL : http://journals.openedition.org/narratologie/6124

DOI : 10.4000/narratologie.6124

ISSN : 1765-307X

Éditeur

LIRCES

Référence électronique

Marc Marti, «Affectivité et sentiment en économie politique : Cartas sobre los obstáculos que la naturaleza, la opinión y las leyes oponen a la felicidad pública du comte de Cabarrús (1795) », Cahiers de Narratologie [En ligne], 18 | 2010, mis en ligne le 15 juillet 2010, consulté le 01 mai 2019. URL : http:// journals.openedition.org/narratologie/6124; DOI : 10.4000/narratologie.6124

Ce document a été généré automatiquement le 1 mai 2019.

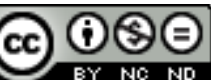

Cahiers de Narratologie - Analyse et théorie narratives est mis à disposition selon les termes de la licence Creative Commons Attribution - Pas d'Utilisation Commerciale - Pas de Modification 4.0 International. 


\title{
Affectivité et sentiment en économie politique : Cartas sobre los obstáculos que la naturaleza, la opinión y las leyes oponen a la felicidad pública du comte de Cabarrús (1795)
}

\author{
Marc Marti
}

1 Longtemps considéré comme un homme pratique et non comme un théoricien, Cabarrús n'en demeure pas moins un représentant original de la Ilustración ${ }^{1}$. Comme le souligne José Antonio Maravall, le fondateur du Banco de San Carlos se distingue par un éclectisme théorique qui, loin de lui être propre, n'est que le reflet des hésitations idéologiques des composantes du despotisme éclairé. Dans une certaine mesure, sa pensée économique se retrouve dans les Cartas sobre los obstáculos que la naturaleza, la opinión y las leyes oponen a la felicidad pública (1795).

2 L'intérêt de cette œuvre réside d'abord dans sa forme. En effet, contrairement aux pratiques déjà établies par les sciences économiques à l'époque (avec quelques spécificités hispaniques qu'il convient de prendre en compte), le texte se présente comme un ensemble de quatre lettres adressées à deux destinataires.

Ensuite, de l'aveu même de l'auteur, l'écriture et la rhétorique qui se mettent en place semblent plus proches du discours littéraire que du discours scientifique. En effet, l'introduction, rédigée en 1808 au moment de la première publication, insiste sur la sensibilité d'esprit et l'imagination au service d'idées exactes, situant ainsi l'œuvre à michemin entre l'imagination, les sentiments et la raison.

Cependant, ces sentiments qui fondent l'analyse économique et politique sont loin d'être une simple rhétorique, ils constituent le fondement d'une pensée qui s'affirme comme l'expression de lois naturelles. 


\section{De l'économie politique dans l'Espagne des Lumières}

\section{L'économie politique, origine et constitution}

5 L'économie politique, considérée comme une branche des sciences économiques, est au départ issue des théories mercantilistes, les premières à envisager l'activité économique d'un point de vue laïc dans la perspective des affaires publiques. Les historiens de l'économie considèrent que cette science commence à émerger au XVI ${ }^{e}$ siècle. Elle essaie de répondre en particulier à l'interrogation que suscite la hausse des prix engendrée par l'arrivée de l'or des Amériques. Au-delà de cette conjoncture, les réflexions portent sur les mesures à adopter à l'échelle nationale afin d'augmenter la richesse de la nation.

Dans le processus de formation, l'histoire du syntagme a de l'importance, tout comme celui du substantif-pivot 'économie'. En français, on fait généralement remonter cette science au traité d'Antoine de Montchrestien Traité d'économie politique (1615). Cependant, les travaux sur le syntagme font apparaitre que son sens contemporain remonte à l'époque des physiocrates et de l'encyclopédie, avec des occurrences nettes dès $1755^{2}$.

7 En castillan, le terme economía est d'abord associé à la bonne administration d'un patrimoine familial dans le premier dictionnaire de l'Académie espagnole de 1732 et jusqu'à l'édition de 1783, qui ne font que reprendre le sens de l'étymon grec $^{3}$ :

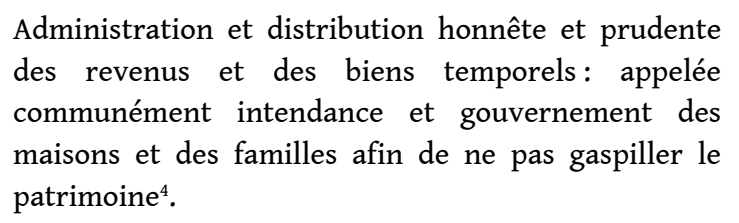

8 À partir de 1791, la référence au patrimoine familial disparait, dans une définition identique à la précédente mais tronquée de la seconde partie, c'est l'« administration honnête et prudente des biens temporels $»^{5}$. Cependant, il faut attendre l'édition de 1817 pour que le syntagme economía política apparaisse. C'est « une science traitant de la richesse des nations, des causes de son augmentation ou de sa diminution ${ }^{6}$ ».

Dans la même édition, le terme economista est défini comme «celui qui comprend exactement les règles et les projets d'économie. Politicae œconomicae peritus ${ }^{7}$ ».

10 L'analyse philologique, complétée par une consultation du CORDE (CORpus Diacrónico del Español), révèle que la première occurrence du syntagme dans son sens contemporain se retrouve deux fois sous la plume de Jovellanos en 1777, dans une lettre à Campomanes. Ce document est d'une grande importance pour la définition de l'économie politique espagnole. En effet, Jovellanos y décrit les textes des arbitristas des XVI ${ }^{e}$ et XVII (Sancho de Moncada, Martínez de la Mata, Álvarez Osorio, Fernández Navarrete) comme des traités d'économie politique, démontrant, si besoin était, que les prémisses de cette science se trouvent dans la péninsule très précocement et qu'elle possède des racines nationales profondes.

\section{L'économie politique dans l'Espagne de la fin du XVIII e siècle}

11 Cependant, l'Espagne de la fin du XVIII e siècle n'a pas légué d'écrits d'économie politique marquants pour la pensée européenne, contrairement à la France ou au Royaume-Uni qui vont, dans une certaine mesure, «inventer» le libéralisme et l'école classique. Cette 
nouvelle science connut pourtant un développement important dans la péninsule et de très nombreux textes témoignent d'un essor qui possède ses propres caractéristiques, tout en étant fortement influencé par l'apport des écrits français et anglais, dont les concepts sont repris avec des sens différents ${ }^{8}$. Globalement, cette pensée espagnole est très pragmatique, car issue d'une solide expérience de terrain. La plupart des auteurs sont des hommes d'État en exercice ou ayant exercé des responsabilités dans l'appareil administratif de la monarchie absolue. Campomanes et Jovellanos sont sans conteste les deux auteurs majeurs d'une période que leurs écrits contribuent à baliser. Le premier est le représentant d'une pensée qui fait de l'État un acteur majeur de la réforme, alors que le second adoptera et adaptera la pensée libérale anglaise à la situation particulière de l'Espagne.

Par ailleurs, et c'est un élément souvent méconnu par les historiens non-hispanistes, l'Espagne à la fin du XVIII siècle est un État doté d'un appareil statistique de tout premier ordre, remarquable pour son époque. En effet, trois grands recensements nationaux $(1768,1787,1797)$ comptabilisent non seulement la population par tranches d'âge, sexes, régions, provinces, villes, bourgs et villages, mais aussi par catégories socioprofessionnelles. La richesse nationale est aussi évaluée en 1746 dans l'opération du Catastro dont l'objectif était une réforme fiscale dans les royaumes de Castille. Enfin, en 1799, une évaluation de la production nationale fut réalisée, préfigurant les calculs actuels de PNB9.

À ces opérations d'envergure nationale, il conviendrait d'ajouter les enquêtes commanditées auprès des fonctionnaires locaux sur des problèmes économiques ponctuels. Ce fut le cas par exemple lorsqu'en 1765 on promulgua le libre commerce des grains en supprimant la taxation. Les rapports des corregidores sur les effets de la mesure (et la situation sociale délicate en certains endroits) amenèrent les gouvernants à abroger le texte ${ }^{10}$.

Globalement donc, l'économie politique espagnole (et l'adjonction d'une épithète de nationalité nous semble pertinent) est le croisement des théories européennes et d'une pensée pragmatique qui s'alimente de l'expérience de terrain et d'un appareil statistique parmi les premiers d'Europe.

\section{La rhétorique à l'œuvre : l'économie politique comme littérature}

En 1983, dans un texte considéré comme fondateur, l'économiste américaine Deirdre Nansen McCloskey a affirmé et démontré la dimension rhétorique des sciences économiques ${ }^{11}$. À la suite de cet article, de nombreux travaux, principalement aux EtatsUnis, se sont intéressés aux éléments constituant le discours économique ${ }^{12}$. Faisant le bilan de deux décennies de controverses suscitées par ce sujet, Ludovic Frobert retient deux directions qui pourraient être profitables. D'abord, il conviendrait de relativiser des concepts tels que ceux de vérité et de réalité, hérités du positivisme sans aucune distance. Ensuite, et c'est le point qui aujourd'hui nous intéresse, il faut se consacrer à «l'étude outillée de la triade auteur-texte-contexte ${ }^{13}$ ». Cette démarche permettrait de s'inscrire dans une nouvelle heuristique, dans le cadre d'une vaste histoire de l'économie politique ${ }^{14}$ . C'est dans cette perspective que se place l'étude que nous proposons des Cartas de Cabarrús et de leur dimension littéraire. 


\section{Une forme de son temps?} amicale ${ }^{15}$. Bien entendu, les circonstances expliquent en partie un choix qui n'est pas uniquement conventionnel. Le texte, constitué de trois longues lettres, était destiné à son ami Gaspar Melchor de Jovellanos, qui venait de rendre public, au nom de la Société Économique des Amis du Pays de Madrid, son Informe en el expediente de la Ley Agraria, un texte très important qui établissait les réformes à adopter pour développer l'agriculture espagnole. C'est en référence à cette œuvre que Cabarrús, alors en disgrâce, propose à son ami de compléter le travail en examinant à son tour les problèmes auxquels est confronté le pays, comme il le précise dans la première lettre sur l'agriculture :

Mon ami, conformément à ce que je vous ai proposé, je vais examiner les trois parties qui composent votre excellent projet de Loi Agraire, c'est-à-dire, les obstacles de la Nature, de l'Opinion et de la Loi ${ }^{16}$. destinée au puissant Godoy, alors Premier Ministre du Roi. Cette dernière lettre, qui est une sorte de longue préface, transforme rétroactivement le statut des trois autres. Le texte, qui au départ était un débat à distance entre deux amis économistes, devient un traité soumis au Prince (en l'occurrence à son plus haut représentant) afin de l'aider dans sa tâche de bonne gouvernance, qui doit impliquer la réforme économique et institutionnelle :

J'ai examiné, dans mes lettres à Jovellanos, ces trois sortes d'obstacles, et les moyens de les surmonter. Maintenant, dans celle-ci [lettre à Godoy], j'ai essayé d'organiser un mode de gouvernement capable de réussir cette entreprise ${ }^{17}$.

Ce faisant, l'auteur accomplit un geste de sociabilité dont la valeur symbolique est grande. Il fait entrer le ministre dans le cercle de ses amis en lui faisant partager des préoccupations communes, avec « franchise, sincérité et confiance ${ }^{18}$ ».

Le texte restera cependant inédit durant le règne de Charles IV, à la fois pour des raisons de personnes (Jovellanos et Godoy connaîtront la disgrâce dès 1797) et pour les idées libérales qu'il développait. Les choses changèrent en 1808, quand Cabarrús accepta d'être ministre des Finances (Hacienda) du nouveau roi d'Espagne, Joseph $1^{\mathrm{er}}$. Cette nomination explique peut-être la publication de l'ensemble, auquel l'auteur ajouta la Memoria al Rey nuestro señor Carlos III para la extinción de la deuda nacional de 1783. En procédant de la sorte, il offrait au public la justification de ses compétences techniques pour un poste aussi élevé. C'était une façon de suggérer que sa nomination ne devait rien au hasard, moins encore aux circonstances politiques troublées, comme il l'expose dans la préface écrite pour la circonstance :

La publication de cette œuvre serait aujourd'hui inutile si on considérait seulement les grandes espérances que la patrie doit concevoir au regard des vertus et des lumières $\mathrm{du}$ Souverain qui nous gouverne, si docte dans l'art de régner qu'il est convaincu que s'il ceint la couronne c'est seulement pour faire le bonheur de ses sujets. Mais comme il a voulu que j'occupe une petite place dans son grand 
projet, je juge qu'il n'est pas inopportun que j'expose franchement les principes que j'ai suivis jusqu'à présent, pour que mes actions y soient rapportées, pour que la censure publique les rectifie ou pour que celui qui sera doté de plus de talent et d'expérience en établisse d'autres afin d'éclairer la nation. Avec le même dessein, je publie aussi un mémoire présenté il y a vingt-cinq ans au roi Charles III sur le crédit public ainsi qu'un système fiscal ${ }^{19}$. la fidélité de son auteur à des principes immuables. Ce faisant, il interdit implicitement de soupçonner son auteur d'opportunisme dans sa collaboration avec le «Roi intrus ». Il prendra soin cependant, dès le début de la préface, d'anticiper les critiques en faisant remarquer que la forme ne correspondait pas à ce que l'on pourrait attendre d'un traité d'économie politique :

Quand j'ai écrit ces lettres pour y exposer mes propres réflexions au sujet de l'administration publique, ses vices et les réformes qui me paraissaient les plus opportunes, j'ai particulièrement soigné l'exactitude des idées sans me soucier des ornements du style, et moins encore de la précision didactique avec laquelle on traite habituellement ce type de sujet. Je me suis laissé conduire par mon imagination, je me suis abandonné à ma sensibilité d'âme, comme le permet une correspondance familière ${ }^{20}$. la définition que Cabarrús donne de ses Cartas, tout en rappelant qu'il n'a pas suivi la norme formelle qu'imposerait le sujet, à savoir la précision didactique et les ornements stylistiques. La préface nous invite donc à considérer ce texte comme une production plus proche de la littérature que du raisonnement. Cependant, cet avertissement peut être lu de plusieurs façons. Il est probable - et nous nous tiendrons à cette idée à titre d'hypothèse - qu'en 1808 , la situation de communication a changé pour l'auteur. La forme épistolaire, qui supposait une manière de s'adresser au Prince depuis une position inférieure, n'est plus adaptée au personnage qu'est devenu Cabarrús, qui peut rencontrer facilement le nouveau souverain dont il est devenu un des proches collaborateurs et avec lequel il entretiendra même une correspondance assidue lors de ses déplacements ${ }^{21}$. Par ailleurs, et c'est une hypothèse qui mériterait confirmation, il est aussi possible que la collection de lettres, marquée par son origine littéraire, soit une forme passée de mode pour cette nouvelle science positive qu'était entre-temps devenue l'économie politique. En effet, à l'exception notable des Cartas económico-políticas attribuées à León de Arroyal ${ }^{22}$, on trouve peu de textes économiques qui empruntent cette configuration. La majorité d'entre eux utilisent les formes consacrées, éprouvées et 'officielles' du mémoire, de la dissertation, du discours, du rapport sur dossier, dont Cabarrús dominait aussi la pratique 23. La forme épistolaire non fictionnelle utilisée pour le thème économique est donc minoritaire et elle laisse la place aux sentiments.

\section{Les sentiments : de la circonstance à la démonstration}

Les sentiments sont d'abord explicables par les circonstances. Le premier sentiment, fortement exprimé dans les propos liminaires, est l'amitié. C'est elle qui unit le scripteur 
avec ses deux lecteurs privilégiés. Jovellanos est l'ami de longue date et Godoy le nouvel ami, auquel l'auteur adresse la première lettre. La différence entre ces deux destinataires est cependant notable, car, si on ne peut douter de la sincère amitié qui liait Jovellanos et Cabarrús (au-delà de leurs affinités idéologiques), l'amitié avec Godoy est très circonstancielle: c'est avant tout une marque de gratitude. Le Prince de la Paix l'a protégé et lui a permis la rédemption après son enfermement au château de Batres.

En sortant du château de Batres, je sentais la
nécessité de soulager mon esprit des méditations
profondes et incessantes qui, dans cet antre de
douleur, l'avaient successivement distrait ou exalté.
Quand, pour m'apaiser, je profitais de l'occasion que
me donnaient mon incomparable ami Jovellanos et
son immortel projet de Loi Agraire, qui aurait dit que
j'allais volontairement communiquer cette effusion
de nos cœurs au principal ministre de la monarchie?
Mais ce ministre a consolé mes malheurs. Il s'est
opposé avec une inflexible constance aux
innombrables intrigues qu'ont essayées de monter,
jusqu'au dernier moment, les auteurs infâmes de
cette proscription, pour s'opposer à la droiture du
Roi. Il a fait triompher ma cause, il a gagné ma
confiance et c'est mon ami. Par ce titre seul, je lui fais
ce gage sacré d'amitié24.

Ce sentiment amical s'exprime au niveau des adresses au lecteur sous la forme amigo mío qui ouvrent ou ferment les démonstrations. C'est lui qui conditionne le style de l'échange, le plaçant dans le registre de la familiarité et du partage de points de vue. À ce titre, le scripteur pourra analyser la réalité tout en intégrant son propre point de vue sentimental. En effet, l'œuvre abonde en marqueurs de subjectivité. Les points d'exclamation et d'interrogation sont présents dans toutes les démonstrations, les constats et les adresses au lecteur. De la même façon, les exclamations du type "oh", «ah» et «ay » ponctuent bon nombre de phrases. Au-delà de ces marques classiques de l'investissement du 'Je' et de l'interlocution, il convient aussi d'analyser les passages où l'analyse de la réalité et l'exposé des réformes constituent des espaces où s'expriment les sentiments.

Un premier exemple de l'implication de l'observateur nous est donné dans la première lettre à Jovellanos, où Cabarrús indique qu'il ne peut contempler l'immense hôpital de Madrid, «sans douleur ni tristesse ${ }^{25}$ ». Un peu plus loin, l'analyse du phénomène des enfants abandonnés (expósitos) l'amène à se demander « de combien de réflexions, toutes aussi douloureuses, ce sujet est-il susceptible ${ }^{26} »$.

Cependant, on aurait tort de penser que ce sont uniquement les circonstances qui expliquent le recours aux sentiments. En effet, bon nombre de démonstrations s'appuient non seulement sur un partage des sentiments entre le scripteur et les lecteurs, auxquels on donne à voir, à travers le prisme sentimental, la réalité ou les solutions à apporter. Les sentiments sont aussi présentés comme des composantes sociales dont il faut tenir compte. 


\section{Les sentiments au service de la réforme}

Cabarrús est en effet convaincu que la réforme des institutions et de l'économie passe par la prise en compte des sentiments qui animent les individus. Le début de la démonstration sur le système d'assistance aux pauvres le rappelle :

Il faut en appeler à ces inclinations primitives de la nature, qui n'a jamais inversé impunément notre fausse sagesse. Nous nous aimons nous-même, nous aimons nos enfants, nos parents, nos voisins, et cet amour diminue à mesure que les objets s'éloignent du centre. Telle est la nature humaine. Par quelle espèce de délire a-t'on tenté de remédier ces affects et de remplacer par des règlements et des employés les inclinations d'un cœur, qui me poussent impérieusement à vêtir la créature innocente que j'ai vue naître et sécher les larmes qui font couler les miennes! Tous les créateurs de fondations ont commis cette erreur, en pensant transférer aux administrateurs et aux employés l'esprit de charité qui les animait ${ }^{27}$.

Dans ce passage, il affirme que le rôle social des individus doit être le plus proche possible de leurs sentiments naturels. Ainsi, dans le passage sur les institutions d'assistance, il récuse les grandes organisations, tenues par des administrateurs, des employés, avant tout gestionnaires, donc incapables de compassion envers les pauvres. Il défend par contre le modèle d'une assistance confiée à des structures à taille humaine, où celui qui donne est proche de celui qui reçoit, ce qui permet de conserver le sentiment.

De la même façon, son projet de repeuplement des campagnes par les élites propriétaires n'est fondée que sur les sentiments :

La vie à laquelle je les incite est celle du bonheur: quand bien même seraient-ils insensibles aux bénédictions qui les attendent, aux larmes d'allégresse, à tous les témoignages de joie et de gratitude publique, le cœur humain ne l'est pas à la vue et à la contemplation de la propriété. Nous palpitons tous de plaisir en considérant le foyer où nous sommes nés, l'arbre qui a protégé de son ombre les jeux de notre enfance, ainsi que ceux que nous avons vus planter, qui ont notre âge, qui ont grandi avec nous et pour lesquels nous ressentons une sorte de sympathie fraternelle. De plus, existe-t-il une seule commodité, un seul plaisir que les grands propriétaires ne puissent atteindre en résidant sur leurs possessions? Un air plus pur, des aliments plus savoureux et plus abondants, des habitations plus spacieuses, plus commodes et décorées bien plus délicieusement ${ }^{28}$.

Cette façon de voir les choses repose sur une théorie du contrat social clairement exposée ${ }^{29}$ :

Mais la société a été formée pour maintenir un juste équilibre entre toutes les passions et les forces individuelles, pour les diriger vers le bonheur 
commun : ainsi s'expliquent la politique et la morale qui sont une seule et même chose. Qui peut douter que la plus intime coopération à l'intérêt général ne produise pas le bonheur personnel et que la vertu et l'amour propre éclairés ne concourent pas au même objectif? propio ilustrado), tirera une grande satisfaction (felicidad personal) de l'accomplissement de l'intérêt général.

\section{Une rhétorique libérale : la force de la Nature}

31

$$
\begin{aligned}
& \text { lettre liminaire adressée à Godoy : } \\
& \qquad \begin{array}{l}
\text { Ne croyez pas qu'il s'agisse d'une critique de tel ou tel } \\
\text { ministre ou de telle ou telle époque. Non, mon ami, } \\
\text { l'ancienneté de l'erreur se perd dans la nuit des } \\
\text { temps. Au premier maillon des abus qui nous } \\
\text { oppriment, d'autres se sont ajoutés, siècle après } \\
\text { siècle, et chaque ministre qui n'a pas eu le courage de } \\
\text { rompre cette chaîne s'est vu obligé à la consolider }{ }^{30} \text {. }
\end{array}
\end{aligned}
$$

La présence des sentiments entre dans le cadre d'une rhétorique plus générale. Pour les libéraux, comme pour Cabarrús tout au long de ses Cartas, il s'agit de démontrer que la réforme n'est qu'un retour aux principes naturels, qui ont été obscurcis par l'empilement de règlements, pratiques et institutions durant le cours de l'histoire du Pays. De ce point de vue, ses conceptions se rattachent aux théories du droit naturel qui s'étaient vulgarisées tout au long du siècle. Cette idée sous-tend la quasi-totalité de l'exposé dans la

La solution proposée à cet état de fait semble évidente : il faut retourner à l'état originel des sociétés politiques afin de retrouver les principes du bon gouvernement
Pour réparer cette négligence de vos prédécesseurs, pour faire plus qu'eux, pour faire la seule chose qui est nécessaire, retournez à l'origine des sociétés politiques et vous verrez disparaître tous les accidents qui aujourd'hui les distinguent ${ }^{31}$.

C'est à ce titre que les sentiments bénéficient d'une place de choix. Le sentiment étant naturel, il faut gouverner en lui rendant sa place. On retrouve là un point de vue largement partagé par les économistes classiques, dont l'intention est souvent de revenir à un mécanisme «naturel ».Ce contexte explique que Cabarrús affirme non seulement la nécessité d'un gouvernement naturel mais aussi (ce qui peut nous surprendre) que la politique doit se faire avec les sentiments :

Non, mon ami, la science du gouvernement n'a pas besoin de doctrines secrètes, ni de grands efforts de l'esprit: elle se trouve dans le cœur de tout honnête homme. S'il étudie la nature en son être comme en ses semblables, il les aimera tendrement et préfèrera leur bonheur à tout le reste, et même au paradis ${ }^{32}$.

La bonne façon de gouverner c'est finalement d'observer les sentiments, qui ne sont qu'une représentation de la Nature, un concept récurrent dans l'idéologie libérale. 


\section{Conclusion : économie politique et sentiments}

\section{NOTES}

1. Voir l'introduction de l'article de J.A. Maravall : «Cabarrús y las ideas de reforma política y social en el siglo XVIII español », Revista de Occidente, XXIII-69, 1968, pp. 273-300.

2. Voir en particulier l'article de Marie-France Piguet, « Economie/Économie (politique) dans le texte informatisé de l'Encyclopédie ", Recherches sur Diderot et sur l'Encyclopédie, numéro 31-32 de l' Encyclopédie en ses nouveaux atours électroniques : vices et vertus du virtuel, [en ligne], mis en ligne 
le 16 mars 2008. URL: http://rde.revues.org/index2983.html. Consulté le 04 mars 2009, pp. 134-135.

3. Voir Marie-Laure Acquier, «Enjeux et ressorts de la prose d'idées dans l'Espagne du XVIIe siècle ", Cahiers de Narratologie, ${ }^{\circ} 14$, mis en ligne le 1 septembre 2008, URL :http://revel.unice.fr/ cnarra/document.html?id=613.

4. Diccionario de la Real Academia Española (DRAE), 1732 : «Administración y dispensación recta y prudente de las rentas y bienes temporales; lo que comúnmente se dice régimen y gobierno de las casas y familias para que no se desperdicie la hacienda ».

5. DRAE, 1791 : «Administración recta y prudente de los bienes temporales ».

6. DRAE, 1817 : «Ciencia que trata de la riqueza de las naciones, y de las causas de su aumento o disminución ».

7. DRAE, 1817: «El que entiende mucho de reglas y proyectos de economía. Politicae œconomicae peritus ».

8. Voir à ce propos notre article sur l'influence d'Adam Smith sur Jovellanos et la façon dont l'Asturien l'a adapté : Marc Marti, «Théories économiques, libéralisme et pensée politique dans El informe en el expediente de la Ley Agraria ", in L'Espagne au XVIIIe siècle. Actes du Colloque international (Saint Étienne, décembre 1997), Saint-Étienne, Université, 1997, pp. 189-204.

9. L'opération est connue sous le nom de Censo de frutos y manufacturas de España.

10. Voir notre travail, «Le libéralisme économique des Lumières, conservatismes et évolutions » in Goya, images de son temps, de l'Espagne des Lumières à l'Espagne libérale, Amiens, Publications de l'université de Picardie, 2005, pp. 47-60.

11. Donald N. McCloskey, "The Rhetoric of Economics ", Journal of Economic Literature, vol. 31, 1983, pp. 482-517.

12. Voir la synthèse proposée par Ludovic Frobert, Donald N. MC Closkey la rhétorique des sciences économiques, Lyon, éditions de l'ENS, 2004.

13. Ludovic Frobert, Ibid, version HAL SHS du texte, URL: http://hal.archives-ouvertes.fr/ docs/00/24/18/43/PDF/2e_introduction.pdf

14. Ludovic Frobert, Ibid, version HAL SHS du texte, URL: http://hal.archives-ouvertes.fr/ docs/00/24/18/43/PDF/2e_introduction.pdf

15. Voir Cécile Mary Trojani, L'écriture de l'amitié dans l'Espagne des Lumières, Toulouse, Presses universitaires du Mirail, 2004.

16. Cartas sobre los obstáculos que la naturaleza, la opinión y las leyes oponen a la felicidad pública, Vitoria, 1808, p. 27 : «Amigo mío : conforme a lo que ofrecí a Vm. voy a recorrer los tres puntos en que divide su excelente proyecto de ley Agraria, esto es, los obstáculos de la naturaleza, los de opinión y los de legislación ». [Toutes les traductions ont été réalisées par nos soins].

17. Cartas, p. $26:$ : He recorrido en mis cartas a Jovellanos estas tres clases de obstáculos, y los medios de dirimirlos, ahora en ésta [carta a Godoy] he tratado de organizar un gobierno capaz de esta grande empresa ".

18. Ces trois termes sont employés par Cécile Mary Trojani pour désigner la sociabilité domestique à travers l'amitié. Op. cit., p. 273.

19. Cartas, pp. 5-6 : « Hoy seria inútil la publicación de esta obra, si sólo hubieran de considerarse las grandes esperanzas que debe la patria concebir de las virtudes e ilustración del Soberano que nos gobierna, tan instruido en el arte de reinar, como persuadido de que sólo para hacer felices a sus vasallos ciñe la corona. Pero habiendo querido que yo tenga una pequeña parte en la ejecución de sus altos designios, no juzgo inoportuno exponer francamente los principios que he seguido hasta aquí : o para que mis acciones se cotejen con ellos, para que la censura pública los rectifique, o establezca otros mejores el que dotado de mayor talento y experiencia quiera ilustrar a la nación. Con el mismo intento publico también una Memoria presentada veinte y cinco años ha al señor Carlos III sobre el crédito público y un sistema de contribuciones ». 
20. Cartas, page 1 du prologue non numéroté : « Cuando escribí estas cartas para exponer en ellas mis propias reflexiones acerca de la administración pública, sus vicios y las reformas que me parecían más oportunas, atendí a la exactitud de las ideas, y cuidé poco de los ornatos del estilo, y mucho menos de la precisión didáctica con que suelen tratarse estas materias : dejé correr mi imaginación, y me entregué a toda la sensibilidad de mi alma, como lo permite una correspondencia familiar ».

21. Une réédition contemporaine de ces échanges entre Joseph 1er en voyage et son ministre été publiée par Díaz Torrejón, Francisco Luis et Sánchez Rodríguez, Alfonso, Cartas josefinas; epistolario de José Bonaparte al Conde de Cabarrús (1808-1810), Séville, Fondation Genesian, 2003.

22. Une édition contemporaine en existe : León Arroyal Cartas político-económicas, Oviedo, Centro de Estudios del siglo XVIII, [1787-1795], 1971.

23. L'édition de 1808 des Cartas est composée aussi de la Memoria al Rey nuestro señor Carlos III para la extinción de la deuda nacional, 1783.

24. Cartas, p. 7 : «Cuando al salir del castillo de Batres sentía la necesidad de aliviar mi espíritu de las profundas y continuas meditaciones que en aquella mansión de dolor le habían sucesivamente distraído o exaltado; cuando aprovechaba para desahogar parte de ellas la ocasión que me suministraba mi incomparable amigo Jovellanos con su inmortal proyecto de Ley Agraria, $\_$quien me hubiera dicho que esta efusión de nuestros corazones la había de comunicar voluntaria y gustosamente al principal ministro de la monarquía ? Pero este ministro ha consolado mis desgracias; ha opuesto una constancia inflexible a las innumerables asechanzas con que hasta el último instante los infames autores de esta proscripción intentaron ofuscar la rectitud del rey; ha hecho triunfar mi justicia ; ha conquistado mi confianza, y es mi amigo. Por este título solo le entrego este sagrado depósito de la amistad [...]».

25. Cartas, p. 36.

26. Cartas, p. $42:$ : Y de cuántas reflexiones, todas igualmente dolorosas es susceptible esta materia ».

27. Cartas, pp. 33-34: «[...] es forzoso apelar a estas inclinaciones primitivas de la naturaleza, que nunca invirtió impunemente nuestra falsa sabiduría. Nos amamos a nosotros mismos, a nuestros hijos, a nuestros parientes, a nuestros vecinos, y este amor va relajando a medida que los objetos se alejan de su centro. Tal es la naturaleza humana. Pues por cual especie de delirio se intentó remediar estos afectos y reemplazar con reglamentos y con empleados los estímulos de mi corazón, que me mueven imperiosamente a vestir la criatura inocente que he visto nacer, y a enjugar las lágrimas que excitan las mías! Han incurrido más o menos en este error todos los autores de tantas fundaciones, que pensaron trasladar a los administradores y dependientes de ellas el espíritu de caridad que los animaba.

28. Cartas, p. 115 : « [...] la vida a que yo los llamo, es la de la felicidad : cuando fuesen insensibles a las bendiciones que los esperan a las lágrimas de gozo, a todos los testimonios de la alegría y de la gratitud pública, el corazón humano no lo es a la vista y a la contemplación de la propiedad. Todos palpitamos de placer al considerar los hogares nativos, el árbol que cubrió con su sombra los juegos de nuestra niñez, y los que vimos plantar, que son de nuestro tiempo, que crecieron con nosotros, y con los cuales tenemos no sé que simpatía fraternal... ¿ Hay además una sola comodidad, un placer y una ventaja de la sociedad, que no puedan alcanzar los grandes propietarios viviendo en sus posesiones? Un ambiente más puro, manjares más sabrosos y abundantes, moradas más extendidas, más cómodas y más deliciosamente adornadas ».

29. Cartas, p. 73 : « [...] la vida a que yo los llamo, es la de la felicidad : cuando fuesen insensibles a las bendiciones que los esperan a las lágrimas de gozo, a todos los testimonios de la alegría y de la gratitud pública, el corazón humano no lo es a la vista y a la contemplación de la propiedad. Todos palpitamos de placer al considerar los hogares nativos, el árbol que cubrió con su sombra los juegos de nuestra niñez, y los que vimos plantar, que son de nuestro tiempo, que crecieron con nosotros, y con los cuales tenemos no sé que simpatía fraternal... ¿ Hay además una sola 
comodidad, un placer y una ventaja de la sociedad, que no puedan alcanzar los grandes propietarios viviendo en sus posesiones? Un ambiente más puro, manjares más sabrosos y abundantes, moradas más extendidas, más cómodas y más deliciosamente adornadas ».

30. Cartas, p. $8:$ : [...] no crea vmd. que ésta sea una crítica de tal ministro o de tal época; no amigo : la antigüedad del error se pierde en la noche de los tiempos. Al primer eslabón de la cadena de abusos que nos oprimen se añadieron otros en cada siglo, y cada ministro que no tuvo el valor de romperla, se vio precisado a fortificarla ».

31. Cartas, p. 9 : «Para reparar este descuido de sus antecesores, para hacer más que todos ellos, para hacer lo único que se necesita, trasládese vmd. al origen de las sociedades políticas, y verá desvanecerse todos los accidentes que hoy las distinguen $»$.

32. Cartas, p. 65 : « No, amigo mío, la ciencia del gobierno no necesita recónditas doctrinas, ni esfuerzos de entendimiento: está en el corazón de un hombre de bien, que estudiando la naturaleza dentro de sí mismo, como en sus semejantes, los ama tiernamente y prefiere la felicidad de ellos a todo, y aun a la gloria misma ».

33. Voir notre travail, «Emblèmes et devises des Sociétés Économiques d'Amis du Pays : Analyse d'un discours d'intention ", Bulletin Hispanique, tome $98, \mathrm{n}^{\circ} 1$, Université de Bordeaux III, janvierjuin 1996, pp. 97-120. Les deux devises sont : «Ardiente aspiro y anhelo al bien de mi patrio suelo (Tudela de la Navarra)»; « Para el bien de la patria (Málaga)».

34. Frédéric Prot, «La place de l'énergie du désir de plaisir dans le discours politique au XVIIIe siècle ", Le(s) plaisir(s) en Espagne (XVIIIe - XXe siècles), études coordonnées par S. Salaün et F. Etienvre, Paris, CREC, 2004, consulté le 16 mars 2009, URL : http://crec.univ-paris3.fr/01-Prot.pdf

\section{RÉSUMÉS}

Le travail qui suit est une étude sur l'émergence de l'économie politique en Espagne et de ses relations avec la rhétorique. Nous insistons en particulier sur le recours aux sentiments dans l'écriture d'une des œuvres de référence du premier libéralisme espagnole les Cartas sobre los obstáculos que la naturaleza, la opinión y las leyes oponen a la felicidad pública du comte de Cabarrús (1795).

El siguiente trabajo estudia la emergencia de la economía política en España y sus relaciones con la retórica. Enfocamos en particular el uso de los sentimientos como procedimiento en la escritura de una obra que es una de las referencias mayores del primer liberalismo español, las Cartas sobre los obstáculos que la naturaleza, la opinión y las leyes oponen a la felicidad pública del conde de Cabarrús (1795).

\section{INDEX}

Index chronologique : XVIIIe siècle

Index géographique : Espagne

Mots-clés : Cabarrús, économie politique, libéralisme, rhétorique, sentiments 


\section{AUTEUR}

MARC MARTI

Spécialiste de l'Espagne du XVIII ${ }^{\mathrm{e}}$ siècle, Marc Marti est directeur du CIRCPLES (Université de Nice-Sophia Antipolis). 